\author{
David B. Stafford Johnson \\ William Breidahl \\ Joel S. Newman \\ Kenneth Devaney \\ Alan Yahanda
}

\section{Extraskeletal mesenchymal chondrosarcoma of the rectus sheath}

D.B. Stafford Johnson, M.S.c., M.R.C.P.I., F.F.R. ( ) W. Breidahl, M.B.B.S.

J.S. Newman, M.D.

Department of Radiology,

University of Michigan Medical Center,

1500 East Medical Center Drive,

Ann Arbor, MI 48109-0030, USA

K. Devaney, M.D.

Department of Pathology,

University of Michigan Medical Center,

Ann Arbor, Michigan, USA

A. Yahanda, M.D.

Department of Surgery,

University of Michigan Medical Center,

Ann Arbor, Michigan, USA

\section{Case report}

A 40-year-old woman presented with a 6-week history of a progressive painless swelling of the lower anterior abdominal wall. Clinical examination revealed a $4 \times 5-\mathrm{cm}$ non-fluctuant, non-tender mass. Ultrasound (US) examination using a linear $7.5 \mathrm{MHz}$ transducer revealed a large solid heterogeneous mass arising from the anterior abdominal wall; the mass contained scattered areas of increased echogenicity with distal shadowing consistent with calcification (Fig. 1). Increased vascularity within the mass was demonstrated on color-flow Doppler. CT demonstrated a $5 \times 6-\mathrm{cm}$ soft tissue mass containing numerous areas of coarse calcification that was intimately related to the rectus muscle (Fig. 2) and extended inferiorly to abut the symphysis pubis. Based on the CT
Abstract Mesenchymal chondrosarcomas (MSCs) are a rare form of chondrosarcoma which usually arise in bone. Extraskeletal chondrosarcomas constitute a minority (14-25\%) of MSCs. We describe the imaging features of an extraskeletal mesenchymal chondrosarcoma that arose from the rectus abdominus muscle.

and US findings, the diagnosis of chondrosarcoma was considered. MR images were obtained for multiplanar evaluation of the mass prior to surgery. The mass appeared isointense to muscle on T1-weighted sequences and exhibited heterogeneous high signal on T2-weighted and STIR sequences. It demonstrated heterogeneous enhancement following intravenous gadolinium administration (Fig. 3). MRI confirmed the origin of the mass from the rectus muscle and demonstrated normal bone marrow in the symphysis pubis. Scattered areas of low signal within the mass on all MR sequences, consistent with calcification, were present (Fig. 4). The patient underwent wide surgical excision of the mass including resection of a portion of the pubic symphysis. Grossly, the mass was solid, rubbery, and rather well demarcated from the adjacent
Key words Mesenchymal chondrosarcoma extraskeletal

soft tissues. Its cut section was graywhite and cut with a gritty texture; the mass was solid without cystic change. Histologic analysis revealed a mesenchymal chondrosarcoma with evidence of microscopic involvement of the periosteum of the pubic symphysis (Figs. 5, 6). In areas of the tumor, some of the cartilaginous islands were calcified.

\section{Discussion}

Mesenchymal chondrosarcoma (MSC), originally described by Lichtenstein and Bernstein [1] in 1959, represents approximately $1 \%$ of all osseous chondrosarcomas. While the majority of MSCs are skeletal tumors, they may also arise in the soft tissues; the first case of extraskeletal mesenchymal chondrosarcoma (ESMC) was described in 1964 [2]. 

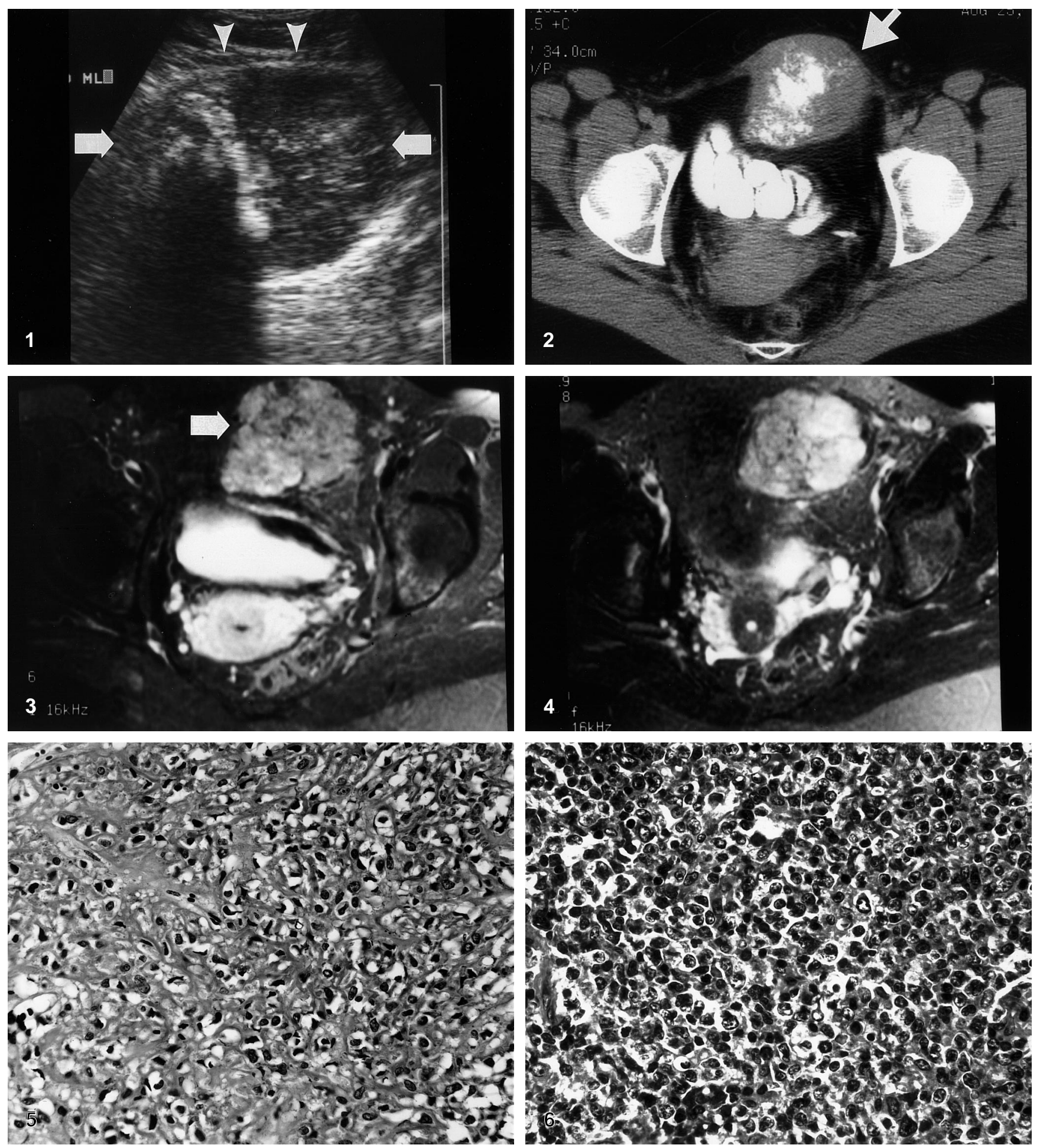

Fig. 1 Longitudinal sonogram of the anterior abdominal wall proximal to the symphysis pubis. There is a well-defined heterogeneous mass (arrows) contiguous with the rectus abdominus muscle (arrowheads). The mass contains central foci with distal acoustic shadowing consistent with calcification
Fig. 2 Axial CT scan above the symphysis pubis. The mass (arrow) extends posteriorly from the rectus abdominal muscle.

Coarse calcifications are present within the mass

Fig. 3 Axial T1-weighted MR image ( TR=500,TE=26) with fat saturation following intravenous administration of gadolinium. The mass (arrow) demonstrates considerable enhancement with irregular areas of low signal interspersed

Fig. 4 Axial T2-weighted MR image $(\mathrm{TR}=3500, \mathrm{TE}=85)$ with fat saturation demonstrates a lobulated, predominantly highsignal mass with intervening low signal intensity foci, some of which appear as septations 
Approximately $14 \%-25 \%$ of MSCs occur in extraskeletal sites [3, 4]. Unlike conventional chondrosarcomas, ESMCs have a female preponderance and occur most frequently in the brain, meninges, and lower extremities [5]. There are two principal age groups in which ESMC occur: those that arise in the central nervous system characteristically occur in the 20- to 30-year age group; ESMCs that arise in soft tissues usually occur in patients older than 40 years of age [6].

There have been few reports of the imaging features of ESMC. In this case, US was the initial radiologic investigation. There has been only one prior case report [7] that detailed the US findings of an ESMC, which also occurred in the rectus sheath. The authors documented the usefulness of US to define the extent of the ESMC and also noted the tumor's relative sonolucency, which was attributed to its myxoid content; this report, however, did not illustrate any histologic findings.

US evaluation of soft tissue neoplasms has previously been reported by several authors; the largest series comprised 50 soft tissue tumors, 14 of which were malignant [8]. Characteristic, but non-specific, features of malignancy included a discrete lesion with ill-defined margins, heterogeneous echotexture, and hypoechogenicity relative to the surrounding tissues. These findings were present in our case. US also demonstrated scattered foci of calcification and blood flow within the mass, which

4 Fig. 5 On medium power examination, the tumor consisted of two components: islands of cartilage, shown here, in which individual tumor cells were distributed throughout a chondroid matrix, and a round cell component, shown in Fig. 6 (hematoxylin and eosin, $\times 175$ )

Fig. 6 On closer scrutiny, the round cell component of the mesenchymal chondrosarcoma consists of a relatively homogeneous population of round to oval cells without striking nuclear pleomorphism or abundant mitotic figures (hematoxylin and eo$\sin , \times 350$ ) excluded a diagnosis of myositis ossificans or hematoma. The largest series of ESMC to date was reported by Shapeero et al. [9] who described the plain radiography, $\mathrm{CT}$, and MR features in seven patients with ESMC. In that series, four of seven cases $(57 \%)$ demonstrated a soft tissue mass with arc, ringed, or stippled calcification on plain radiography.

On CT, a soft tissue mass with predominantly peripheral enhancement following intravenous contrast was evident, which contained focal low attenuation areas possibly representing necrosis. Coarse calcification on CT was demonstrated in four of six patients $(67 \%)$. In our case, extensive calcification was demonstrated on the non-contrast scan; the mass exhibited generalized enhancement following the administration of contrast.

MR imaging may help to distinguish between various types of cartilaginous tumor. The signal intensity of cartilaginous tumors is dependent on their cellular content [10]. The more cellular cartilaginous tumors, such as chondroblastomas or clearcell chondrosarcomas, demonstrate an amorphous configuration that is isointense or hypointense to muscle on T2-weighted images. Other cartilaginous tumors, such as enchondromas and well-differentiated chondrosarcomas, which are less cellular tumors, display a homogeneous high signal intensity on T2-weighted sequences that is thought to reflect the hyaline cartilage with its low cellularity and high water content. These hyaline cartilaginous tumors also exhibit a lobular configuration divided by low-intensity septae consisting of fibrous tissue. A recent study attempted to characterize cartilaginous tumors by means of their enhancement patterns following gadolinium administration. Maartje et al. [11] demonstrated that low grade chondrosarcomas characteristically exhibit septal enhancement corresponding to fibrovascular septation between the lobules of hyaline cartilage. High-grade chondrosarcomas showed inhomogeneous or homogeneous enhancement corresponding to highly cellular areas on histologic analysis. MR performed in four patients in the series reported by Shapeero showed ESMC as a soft tissue mass isointense with muscle on T1weighted sequences with high signal on T2-weighted and proton density sequences; two of four cases in this study exhibited enhancement with gadolinium. In our case, the tumor exhibited gadolinium enhancement in an inhomogeneous fashion; there was also evidence of lobulation. The high signal of ESMC on T2-weighted sequences may reflect foci of cartilage and/or undifferentiated mesenchymal cellular component. ESMCs in general have few cartilaginous elements with a predominance of the mesenchymal component.

The histopathologic differentiation of MSC from malignant hemangiopericytoma and chondrosarcoma may be difficult [12]. The presence of diffusely distributed islands of cartilage with a low-grade cytological appearance throughout the tumor in association with an undifferentiated round to oval cellular component is characteristic of MSC.

Hemangiopericytomas are composed of a dense network of vascular structures about which are arrayed round to oval tumor cells; lacking in hemangiopericytomas, however, are the cartilaginous islands that typify the MSC.

Treatment of ESMC consists of wide surgical excision; the role of adjuvant chemotherapy and radiotherapy is uncertain. In a review of 111 cases by Nakashima et al. [13], no difference in survival was found between bone and soft tissue mesenchymal chondrosarcomas. The mean survival rate in 35 patients in a study by Huvos et al. was 37.9 months [4] . Frequent and long-term follow up is necessary if local recurrence is to be detected at an early stage. Local recurrence is common and usually precedes metastatic disease in the lungs. 


\section{References}

1. Lichtenstein L, Bernstein D. Unusual benign and malignant chondroid tumors of bone. Cancer 1959; 12:1142-1147.

2. Dowling EA. Mesenchymal chondrosarcoma. J Bone Joint Surg 1964; 46:747-754.

3. Guccion J, Font R, Enzinger F, Zimmerman L. Extraskeletal mesenchymal chondrosarcoma. Arch Pathol 1973; 95:336-340.

4. Huvos AG, Rosen G, Dabska M. Mesenchymal chondrosarcoma. A clinicopathological analysis of 35 patients with emphasis on treatment. Cancer $1983 ; 51: 1230-1237$.
5. Wu KK, Collon DJ, Guise ER. Extraosseous chondrosarcoma: report of five cases and review of the literature. $\mathbf{J}$ Bone Joint Surg Am 1980; 62:189-194

6. Louvet C, Gramant A, Krulik M, Jagueux M, Hubert D, Brissaud P, Sirinell A, Augereau B, Tubiana J, Debray J. Extraskeletal mesenchymal chondrosarcoma: case report and review of the literature. J Clin Oncol 1985; 3:858-863.

7. O'Malley BP, Qizilbash AH. Mesenchymal chondrosarcoma of the rectus sheath: case report with ultrasonic findings. J Clin Ultrasound 1977; 5:348-349.

8. Lange TA, Austin CW, Seibert JJ, Angtuaco TL, Yandow DR. Ultrasound imaging as a screening study for malignant soft tissue tumours. J Bone Joint Surg Am 1987; 69:100-105.

9. Shapeero D, Vanel D, Couanet D, Contesso G, Ackerman L. Extraskeletal mesenchymal chondrosarcoma. Radiology $1993 ; 186: 819-826$
10. Cohen EK, Kressel HY, Frank TS, Fallon M, Burk DL, Dalinka MK, Schiebler ML. Hyaline cartilage-origin bone and soft-tissue neoplasms: MR appearance and histologic correlation. Radiology 1988; 167:477-481.

11. Maartje JA, Bloem JL, Eulderink F, Hogendoorn PC, Taminiau AHM. Cartilaginous tumors: correlation of gadolinium-enhanced MR imaging and histopathological findings. Radiology 1993; 186:813-817.

12. Bertoni F, Picci P, Bacchini P, Capanna $\mathrm{R}$, Innao V, Bacci G, Campanacci M. Mesenchymal chondrosarcoma of bone and soft tissues. Cancer 1983; 52:533-541.

13. Nakashima Y, Unni KK, Shives T, Swee RG, Dahlin DC. Mesenchymal chondrosarcoma of bone and soft tissues. Cancer 1986; 57:2444-2452.

\section{ANNOUNCEMENTS}

European Spine Society

8th Annual Meeting

September 10-13, 1997

Kos, Hellas, Greece

For further information please contact:

E.P. Velikas, M.D.

(Organizing Committee),

18 Ipsilandou Str.-Kolonaki,

GR-106 76 Athens, Greece.

Tel. (+30)1-7249050, Fax (+30)1-7241003

or J. Reichert Schild (Scientific Secretariat),

Seefeldstrasse 16

CH-8610 Uster, Switzerland.

Tel. (+41)1-9941404, Fax (+41)1-9941403.

\section{0th Annual Course}

Radiology 1997 - Thoraco-Abdominal Imaging

September 11-13, 1997

Minneapolis, MN, USA

Fee: $\$ 395$.

For further information please contact: Office of Continuing Medical Education, University of Minnesota, Suite 107, 615 Washington Avenue S.E. Minneapolis, MN 55414,

Tel.: (612)626-7600

or Toll Free 1-800-776-8636,

Fax: (612)626-7766,

Internet: http://www.cee.umn.edu:80/cme/

World Congress on Medical Physics

and Biomedical Engineering

September 14-19, 1997

Nice, France

For further information please contact:

Nice97, See, General Secretariat,

48 rue de la Procession,

F-75724 Paris Cedex 15, France.

Tel.: +331444960 60,

Fax +33144496044

e-mail: nice97@univ-paris12.fr.

\section{2nd Interventional MRI Symposium}

October 17-18, 1997

Düsseldorf, Germany

For further information please contact:

PD Dr. Thomas Kahn,

Institut für Diagnostische Radiologie,

Heinrich-Heine-Universität,

Moorenstrasse 5 .

D-40225 Düsseldorf, Germany.

Tel. +49-(0)2 11-8 11-77 52/87 67,

Fax +49-(0)2 11-8 11-61 45,

e-mail: kahn@mr.uni-duesseldorf.de,

http://www.mr.uni-duesseldorf.de
VII Alex Norman Lectureship Series

in Radiology: Advanced Musculoskeletal Imaging - The Shoulder

December 9, 1997

New York, NY, USA

Sponsored by: Hospital for Joint Disease, New York, NY, New York University School of Medicine. Faculty: D. Resnick, V. Chandnani, J. Beltran, J. Brown,

N. Schoenberg, J. Zuckerman, A. Rokito,

D. Rose, F. Cuomo, M. Rafü.

Registration fee: Physicians \$100,

Residents/Technologists \$50.

For further information please contact:

MS. Cathy Smith,

Tel.: (212)598-6373,

Fax: (212)598-6125 